\title{
The Amygdala Is Essential for the Development of Neuronal Plasticity in the Medial Geniculate Nucleus during Auditory Fear Conditioning in Rats
}

\author{
Stephen Maren, Stanley A. Yap, and Ki A. Goosens \\ Department of Psychology and Neuroscience Program, University of Michigan, Ann Arbor, Michigan 48109-1109
}

The medial geniculate nucleus of the thalamus (MGN) and the basolateral complex of the amygdala (BLA) are critical components of the neural circuit that mediates auditory fear conditioning. Several studies indicate that neurons in both the MGN and BLA exhibit associative plasticity of spike firing during auditory fear conditioning. In the present study, we examined whether the development of plasticity in the MGN requires the BLA. Single units were recorded from chronic multichannel electrodes implanted in the medial division of the MGN of conscious and freely moving rats. Rats received auditory fear conditioning trials, which consisted of a white-noise conditional stimulus (CS) and a co-terminating footshock unconditional stimulus (US). Unpaired (sensitization) controls received the same number of trials as paired animals, but the CS and US were explicitly unpaired. Before fear conditioning, rats received either an intra-amygdala infusion of muscimol, a GABA recep- $^{-}$ tor agonist, to inactivate BLA neurons or an infusion of the saline vehicle. Auditory fear conditioning produced a substantial increase in both CS-elicited spike firing in the MGN and conditional freezing behavior in vehicle-treated rats receiving paired training. Muscimol inactivation of the BLA severely attenuated the development of both conditioning-related increases in CS-elicited spike firing in the MGN and conditional freezing to the auditory CS. Unpaired training did not yield increases in either CS-elicited spike firing or freezing to the tone $\mathrm{CS}$. These results reveal that the BLA is essential to the development of plasticity in the auditory thalamus during fear conditioning.

Key words: thalamus; basolateral amygdala; single unit; neuronal plasticity; fear conditioning; freezing; rat
Considerable interest in the neural mechanisms of Pavlovian fear conditioning has emerged in recent years. During fear conditioning, rats learn that an innocuous stimulus (the conditional stimulus or CS), such as a tone, predicts the occurrence of an aversive stimulus (the unconditional stimulus or US), such as a footshock. Conditional fear is manifested by various behavioral responses, including freezing. Several lines of evidence indicate that neurons in the basolateral complex of the amygdala (BLA) are essential for fear conditioning (Davis, 1994; Fanselow and LeDoux, 1999; Maren, 1999a; LeDoux, 2000). These and other findings have led to the proposal that the BLA is a critical locus for the encoding and long-term storage of the CS-US associations that underlie fear conditioning.

An alternative possibility is that the essential neuronal plasticity underlying fear conditioning develops in a brain structure afferent (or efferent) to the amygdala (Cahill et al., 1999). By this view, the amygdala plays a role in modulating memory storage and translating fear memories into behavioral responses, such as freezing, but is not a locus for memory storage (Weinberger, 1998; Cahill et al., 1999; McGaugh, 2000). In the case of auditory fear conditioning, it has been suggested that the medial geniculate nucleus of the thalamus (MGN), which transmits auditory information to the amygdala (LeDoux et al., 1990), is a critical locus of

\footnotetext{
Received Oct. 26, 2000; revised Jan. 12, 2001; accepted Jan. 16, 2001.

This work was supported by grants from the National Institute of Mental Health (R29MH57865) to S.M. K.A.G. is a Howard Hughes predoctoral fellow. We thank two anonymous reviewers for helpful suggestions on an earlier version of this manuscript.

Correspondence should be addressed to Stephen Maren, Department of Psychology, University of Michigan, 525 E. University Avenue, Ann Arbor, MI 48109-1109. E-mail:maren@umich.edu.

Copyright $(\subset 2001$ Society for Neuroscience $0270-6474 / 01 / 210001-\bullet \$ 15.00 / 0$
}

neuronal plasticity underlying fear conditioning to acoustic stimuli (Weinberger, 1998). Consistent with this view, associative neuronal plasticity develops in the MGN during aversive conditioning (Gabriel et al., 1975; Supple and Kapp, 1989; Edeline and Weinberger, 1992). Furthermore, MGN neurons exhibit longterm potentiation (Gerren and Weinberger, 1983), and synaptic plasticity occurs in the MGN during fear conditioning (McEchron et al., 1996). Moreover, the latency of conditioning-related increases in BLA spike firing is consistent with a thalamic origin (Quirk et al., 1995, 1997; Maren, 2000). Hence, it is possible that CS-US associations are encoded in the MGN and transmitted to the BLA for the generation of conditional fear responses.

If the necessary CS and US convergence underlying auditory fear conditioning occurs in the MGN (or at any point afferent to the amygdala), then the development of associative neuronal plasticity in the MGN should be independent of the BLA. Poremba and Gabriel (2001) recently examined this possibility in an instrumental learning task. They reversibly inactivated the

This article is published in The Journal of Neuroscience, Rapid Communications Section, which publishes brief, peerreviewed papers online, not in print. Rapid Communications are posted online approximately one month earlier than they would appear if printed. They are listed in the Table of Contents of the next open issue of JNeurosci. Cite this article as: JNeurosci, 2001, 21:RC135 (1-6). The publication date is the date of posting online at www.jneurosci.org.

http://www.jneurosci.org/cgi/content/full/5107 
amygdala with muscimol, a $\mathrm{GABA}_{\mathrm{A}}$ receptor agonist, and recorded multiple-unit activity in the MGN during discriminative avoidance conditioning in rabbits. They found that amygdala inactivation before avoidance conditioning prevented the development of discriminative neuronal firing (greater discharges to a $\mathrm{CS}+$ than a $\mathrm{CS}-$ ) in the MGN. These results reveal that the development of neuronal plasticity in the MGN is dependent on the BLA, at least during instrumental avoidance learning. However, the role of the amygdala in the development of thalamic neuronal plasticity during Pavlovian fear conditioning is still unknown. Therefore, in the present study, we used single-unit recording techniques coupled with muscimol inactivation of the BLA to determine whether the BLA is essential for the development of associative neuronal plasticity in the MGN during auditory fear conditioning in rats.

\section{MATERIALS AND METHODS}

The subjects were 41 adult male Long-Evans rats (200-450 gm) obtained from a commercial supplier (Harlan Sprague Dawley, Indianapolis, IN). The rats were housed individually in Plexiglas cages on a 14/10 hr light/dark cycle (lights on at 7:00 A.M.), provided ad libitum access to food and water, and handled daily. Fourteen rats were used for singleunit recording in the MGN. They were assigned to one of three groups: vehicle paired (VEH-Pair, $n=6$ ), muscimol paired (MUS-Pair, $n=5$ ), and vehicle sensitization (VEH-Sens, $n=3$ ). These rats received intraamygdala infusions of either saline or muscimol before the training session, which consisted of either paired or unpaired CS and US presentations. Three additional rats were used in a control electrophysiology experiment, and the remaining rats $(n=24)$ were used in a behavioral experiment; these experiments are described in Results.

Before behavioral testing, rats were anesthetized with Nembutal and implanted with bilateral guide cannulas (stainless steel, 26 gauge) aimed at the BLA $(2.3 \mathrm{~mm}$ posterior and $5.0 \mathrm{~mm}$ lateral to bregma; $6.3 \mathrm{~mm}$ ventral to dura) and a unilateral recording probe in the MGN $(6.0 \mathrm{~mm}$ posterior and $3.1 \mathrm{~mm}$ lateral to bregma; $5.5 \mathrm{~mm}$ ventral to dura). The recording probe consisted of a twisted bundle of eight tungsten wires (25 $\mu \mathrm{M}$ diameter, $100-200 \mathrm{k} \Omega$ impedance) that extended $1 \mathrm{~mm}$ beyond the tip of a 28 gauge guide cannula. It was aimed at the medial division of the MGN (MGm) and positioned by monitoring auditory-evoked single-unit discharges. Once in place, the recording assembly and cannulas were affixed to the skull with dental acrylic.

After a $3 \mathrm{~d}$ recovery from surgery, the rats began the first of three sessions, which were conducted over a $2 \mathrm{~d}$ period. These sessions consisted of pretraining (day 1, morning), training (day 1, afternoon), and posttraining (day 2, morning). Before the pretraining session, the rats received an intra-amygdala inf usion of sterile saline $(0.9 \%)$ via stainless steel injectors ( 30 gauge) that extended $1 \mathrm{~mm}$ below the tip of the guide cannulas. The injectors were connected to Hamilton syringes with polyethylene tubing, and the syringes were mounted in an infusion pump (Harvard Apparatus, South Natick, MA). Infusions were made at the rate of $0.1 \mu \mathrm{l} / \mathrm{min}$ for $2.5 \mathrm{~min}$, and $1 \mathrm{~min}$ was allowed for diff usion before the injectors were removed.

Twenty minutes after the infusion, the rats were transported to the conditioning chambers $(30 \times 24 \times 40 \mathrm{~cm})($ MED-Associates, Burlington, VT), which were equipped for the delivery of both auditory CSs (whitenoise, $85 \mathrm{~dB}, 2 \mathrm{sec})$ and footshock USs $(1.0-\mathrm{mA}, 0.5 \mathrm{sec})$. A headstage preamplifier (eight op-amps in a source-follower configuration) was connected to the assembly on each rat's head. The headstage cable was connected to a commutator, which permitted the rats to move freely within the conditioning chamber. The rats were then presented with 10 white-noise stimuli [60 sec interstimulus interval (ISI)] to obtain a profile of white-noise-elicited spike firing in the MGN. We used short $(2 \mathrm{sec})$ auditory CSs to replicate previous studies of amygdaloid neuronal firing during fear conditioning (Quirk et al., 1995; Maren, 2000). Neuronal signals were amplified $(10,000 \times)$, filtered $(0.6-6 \mathrm{kHz})$, and digitized $(32$ $\mathrm{kHz}$ per channel) using Experimenter's Workbench software (DataWave Technologies, Longmont, $\mathrm{CO}$ ) during a $3 \mathrm{sec}$ period ( $0.5 \mathrm{sec}$ before, 2 $\mathrm{sec}$ during, and $0.5 \mathrm{sec}$ after each white-noise stimulus). Freezing behavior was continuously acquired during both the white-noise stimuli and the ISIs using an automated system (Maren, 1999b). We used freezing during the $60 \mathrm{sec}$ ISI that followed each CS presentation as a measure of conditional fear of the CS. Thus, freezing was assessed during extinction in the posttraining session.

Six hours after pretraining, the rats received a training session that consisted of five noise-footshock trials. Twenty minutes before training, the rats received either a saline infusion (VEH-Pair, VEH-Sens) or an infusion of muscimol $(0.25 \mu \mathrm{g}$ per side; MUS-Pair). After the infusion, the rats were returned to the conditioning chambers for either "paired" or "unpaired" presentations of the white-noise CS and footshock US. For rats in the paired groups (VEH-Pair, MUS-Pair), the footshock US co-terminated with the white-noise CS, whereas the white-noise CS and footshock US were explicitly unpaired for rats in the "sensitization" group (VEH-Sens; the US occurred either 15, 30 or $45 \mathrm{sec}$ before CS onset on any given trial, and this sequence was varied pseudorandomly). Neuronal data were not recorded during the conditioning sessions. The following day, the rats again received an intra-amygdala saline infusion and were placed in the conditioning chambers $20 \mathrm{~min}$ after the infusion for the posttraining recording session. The posttraining session was identical to the pretraining session and permitted an assessment of white-noise-elicited spike firing in the MGN after training the day before. Freezing data were collected as described for the pretraining session. Our fear conditioning and testing procedures, which incorporated subtle training-to-testing context shifts (time of day, presence of headstage cable), yielded negligible levels of contextual freezing in both the VEH-Pair and VEH-Sens rats.

Neuronal data collected during the pretraining and posttraining sessions were analyzed off-line using Experimenter's Workbench and Autocut software (DataWave Technologies). Single units were isolated on each recording channel using window discriminators and spike clustering algorithms. Autocorrelograms and cross-correlograms and interspike interval histograms were used to verify that isolated single-unit waveforms on each recording channel were generated by single MGN neurons. Cluster boundaries were computed from the posttraining recording session and applied to the pretraining recording session. Units that were not stable over the $2 \mathrm{~d}$ recording period were excluded. All unit data were binned $(50 \mathrm{msec})$ and normalized to the $500 \mathrm{msec}$ pre-CS baseline. For each recording session, peristimulus time histograms (PSTHs) were summed over the 10 white-noise trials, and an average PSTH was computed for all units in each group of rats. The freezing data for each session were transformed to a percentage of total observations. Neuronal and behavioral data were analyzed using ANOVA. For the former, the data from individual neurons (rather than the average neuronal activity for each animal) were analyzed. Post hoc comparisons in the form of Fisher least significant difference tests were performed after a significant omnibus $F$-ratio. All data are represented as means \pm the SEMs.

After behavioral testing, a marking lesion was made at the tip of one electrode by passing anodal current $(80-\mu \mathrm{A}, 10 \mathrm{sec})$. The rats were perfused, and the brain was frozen and sectioned on a cryostat. The sections were stained with $0.25 \%$ thionin to visualize neuronal cell bodies and the cannula tracks. Cannula placements were verified by reconstructing the cannula tracks on stereotaxic atlas templates. Electrode placements were verified using the marking lesions.

\section{RESULTS}

\section{Electrode and cannula placements}

Recording electrode placements in the MGN are shown in Figure 1. Thirteen of the 14 rats had accurate placements in the MGN. One placement (MUS-Pair group) was medial to the MGN; this rat was excluded from the analysis. Most of the electrode placements were located in MGm (10 of 13), and there were no group differences in the location of electrodes within the MGN. Two electrodes were located in the suprageniculate nucleus (SG), and one electrode was located in the ventral division of MGN (MGv). Both the MGm and the SG receive convergent CS and US information and project to the amygdala; the MGv does not receive somatosensory information and does not project directly to the amygdala (LeDoux et al., 1990). BLA cannulas were accurately placed in all rats (data not shown).

\section{Spike firing and behavior}

We recorded from a total of 296 MGN neurons in the 13 rats included in the analysis. Of these, 101 cells $(34.1 \%)$ were found to 

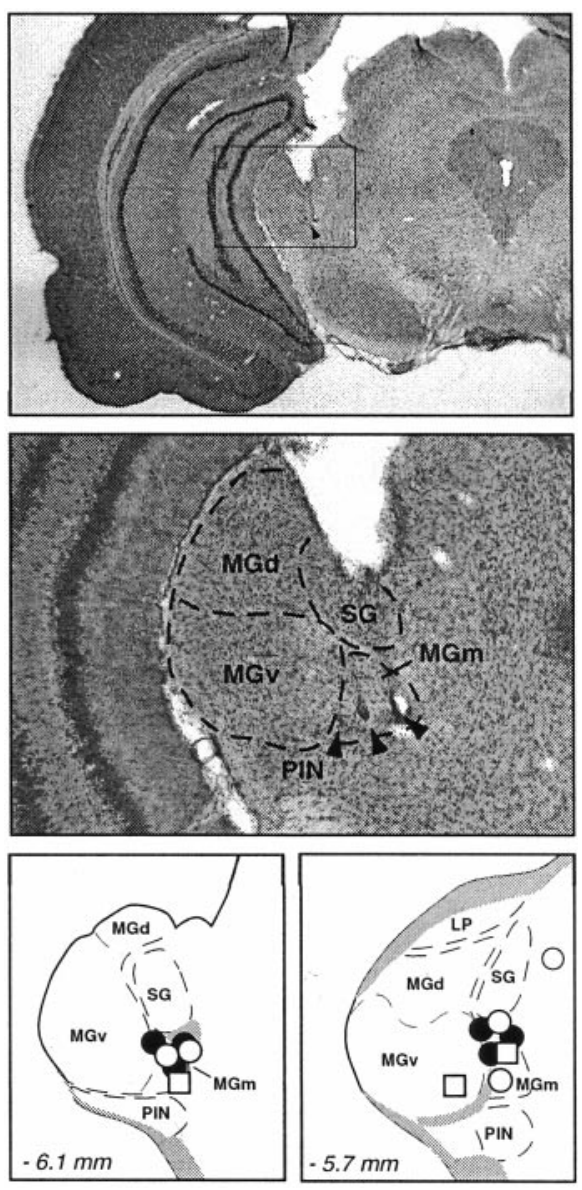

Figure 1. Recording electrode placements in the MGN. Top, Photomicrograph of a thionin-stained coronal section from a representative rat in the VEH-Pair group. The arrowheads indicate the position of three recording wires in the medial division of the MGN. Bottom, Schematic representation of electrode placements in the MGN: VEH-Pair (-), MUS-Pair $(\bigcirc)$, and VEH-Sens $(\square)$. The rat with the electrode placed outside the MGN in the MUS-Pair group was excluded from the analysis. Stereotaxic templates are from Swanson (1998). $M G d, M G m, M G v$, Dorsal, medial, and ventral division of MGN; $S G$, suprageniculate nucleus; $L P$, lateral posterior nucleus; PIN, posterior intralaminar nucleus.

exhibit onset responses to the white-noise stimulus before conditioning. That is, the firing rate of these "onset" cells was at least 3 SDs greater than that in the prestimulus baseline within $50 \mathrm{msec}$ of white-noise onset. As has been reported previously (Bordi and LeDoux, 1994), we observed two classes of auditory-responsive neurons in the MGN. "Transient" neurons ( $n=77 ; 76.2 \%)$ exhibited a phasic increase in spike firing within $50 \mathrm{msec}$ of white-noise onset, and this phasic response returned to baseline levels thereafter. "Sustained" neurons $(n=24 ; 23.8 \%)$ also showed a phasic increase in spike firing but maintained this activation during the entire duration of the $2 \mathrm{sec}$ stimulus. A subset of the transient cells $(n=15 ; 14.9 \%)$ also exhibited pronounced responses to the offset of the white-noise stimulus. The relative proportion of transient and sustained cells that we observed in the present study accords well with other descriptions of auditory-responsive cells in the MGN (Bordi and LeDoux, 1994). In addition to cells that were responsive to the white-noise before conditioning, 65 neurons became responsive to the whitenoise CS after conditioning. Thus, a total of 166 neurons exhibited a short-latency auditory response to the white-noise CS at some point in the experiment.
For the purposes of analyzing the influence of amygdala inactivation on the development of CS-elicited spike firing in the MGN, we restricted our analysis to short-latency auditoryresponsive neurons. These cells were distributed in the experimental groups as follows: VEH-Pair (71 cells, six rats), MUS-Pair (56 cells, five rats), and VEH-Sens (39 cells, three rats). The average firing rate of these neurons was $12.4 \pm 0.9 \mathrm{~Hz}$, and there were no group differences in firing rate $\left[F_{(2,163)}=0.6\right]$. There were also no group differences in the proportion of transient and sustained cells, and these cells exhibited similar conditioningrelated changes. We therefore collapsed these cell types in the analysis.

The average $z$-scores for all of the auditory-responsive cells in each group and recording session are shown in Figure 2. Inspection of Figure 2 reveals that auditory fear conditioning produced robust increases in CS-elicited spike firing in the VEH-Pair group, but not in either the MUS-Pair or VEH-Sens groups. This observation was confirmed in an ANOVA performed on the average unit activity evoked during the $2 \mathrm{sec}$ CS (Fig. $3 A$ ) [group $\times$ session, $F_{(2,163)}=17.7, p<0.0001$ ]. Post hoc comparisons $(p<0.05)$ revealed that cells in both the VEH-Pair and MUS-Pair exhibited increases in firing after fear conditioning, unlike cells in the VEH-Sens group. However, the magnitude of these conditioning-related increases in spike firing were significantly greater in the VEH-Pair compared with the MUS-Pair group. An analysis of the short-latency post-CS onset bin (0-50 msec after CS onset) did not reveal a significant interaction of group and session $\left[F_{(2,163)}=1.9\right]$. However, an analysis of simple effects revealed that only neurons in the VEH-Pair group exhibited conditioning-related increases in spike firing $\left[F_{(1,70)}=8.2\right.$, $p<0.01]$; cells in the MUS-Pair $\left[F_{(1,55)}=1.4\right]$ and VEH-Sens $\left[F_{(1,38)}<1\right]$ groups did not show significant increases in shortlatency CS-elicited spike firing after fear conditioning. Because there was a trend toward an increase in short-latency spike firing in the MUS-Pair group, we further analyzed the first $50 \mathrm{msec}$ of the CS in shorter, $10 \mathrm{msec}$ bins (data not shown). Again, only the VEH-Pair group exhibited significant increases in CS-elicited spike firing (10-50 msec after CS onset) after fear conditioning. Thus, muscimol inactivation of the amygdala blocked the development of associative neuronal plasticity at short latencies after CS onset $(0-50 \mathrm{msec})$ and severely attenuated the plasticity expressed during the $2 \mathrm{sec}$ duration of the CS.

It is possible that intra-amygdala muscimol infusions disrupted thalamic plasticity by directly inhibiting thalamic neurons (as a result of diffusion, for example). We ran three additional control rats ( 72 cells) to examine this possibility. The rats were implanted with electrodes and cannulas as described above; histological analysis verified that the electrodes were placed in $\mathrm{MGm}$. We collected $103 \mathrm{sec}$ epochs (60 sec interval between epochs) of spontaneous MGN activity after the infusion of saline into the BLA and, after $6 \mathrm{hr}$, after the infusion of muscimol into the BLA. Intra-amygdala muscimol infusions did not affect the spontaneous firing rate of MGN neurons [pre-muscimol, $12.0 \pm 1.1$ $\mathrm{Hz}$; post-muscimol, $\left.12.9 \pm 1.1 \mathrm{~Hz} ; F_{(1,71)}=1.1\right]$. This indicates that the effect of amygdala inactivation on associative plasticity in the MGN was not mediated by a direct inhibitory influence of muscimol on MGN spike firing.

In addition to MGN unit activity, we assessed freezing behavior during the pretraining and posttraining sessions. As expected, intra-amygdala muscimol infusion before training blocked the acquisition of conditional freezing (Fig. $3 B$ ) $\left[F_{(2,10)}=6.6, p<\right.$ 0.02]. Post hoc comparisons $(p<0.05)$ revealed that neither 

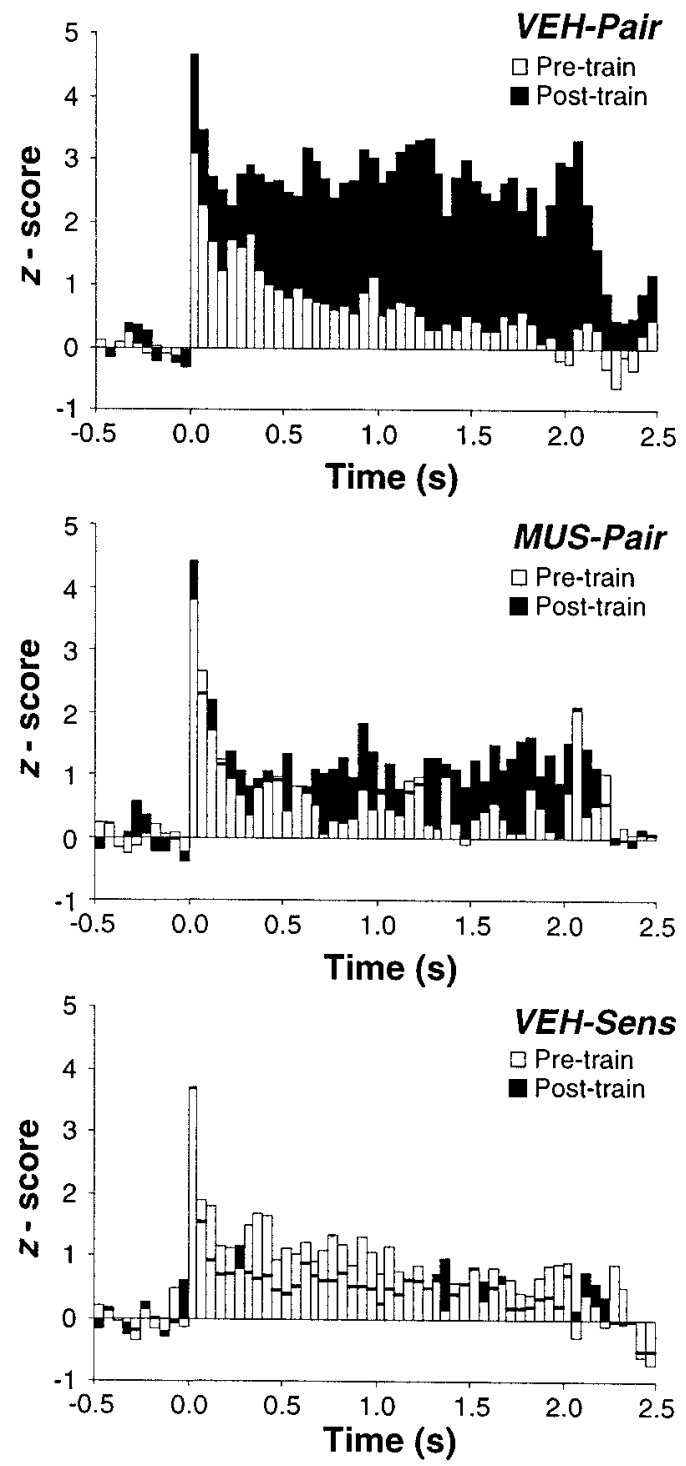

Figure 2. Population peristimulus time histograms of CS-elicited spike firing in the MGN. Mean peristimulus time histograms (spikes summed over 10 trials per session and normalized to the pre-CS baseline; $50 \mathrm{msec}$ bins) for the two recording sessions (Pre-train, open bars; Post-train, filled bars). The histograms represent an average of all auditory-responsive cells in the MGN that were recorded from rats in the VEH-Pair, MUS-Pair, and VEH-Sens groups.

VEH-Sens nor MUS-Pair rats exhibited an increase in conditional freezing to the white-noise CS after fear conditioning; only the VEH-Pair rats exhibited CS-elicited freezing behavior. Thus, muscimol inactivation of the BLA severely attenuated the development of both conditional spike firing in the MGN and conditional freezing behavior, and produced a pattern of spike firing and behavior similar to that of rats receiving explicitly unpaired CS and US presentations.

Although muscimol inactivation of the BLA produced a robust impairment in the development of conditional unit activity in MGN, there was greater conditional unit activity in MUS-Pair cells compared with VEH-Sens cells after fear conditioning (Fig. $3 A$ ). This suggests that MUS-Pair rats may possess a spared memory for auditory fear conditioning that is not expressed on the posttraining test. To examine this issue, we examined whether MUS-Pair and VEH-Sens rats reacquire conditional freezing at
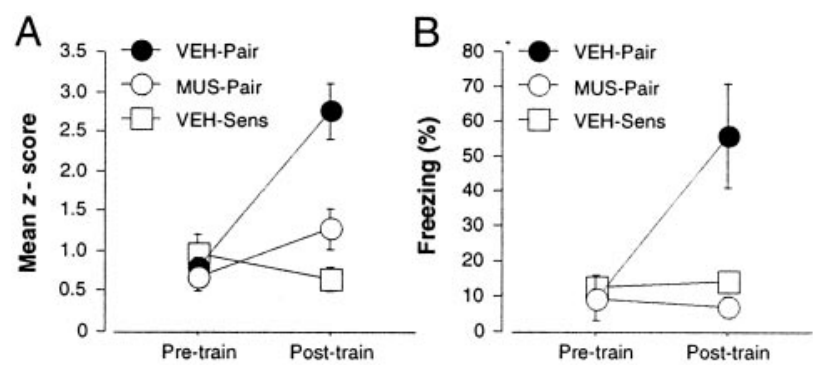

Figure 3. CS-elicited spike firing in MGN and conditional freezing behavior. $A$, Mean $( \pm \mathrm{SEM}) z$-scores during the $2 \mathrm{sec}$ white-noise CS in the pretraining and posttraining recording sessions in rats in the VEHPair (๑), MUS-Pair $(\bigcirc)$, and VEH-Sens $(\square)$ groups. $B$, Mean $( \pm$ SEM) percentage of freezing to the white-noise CS during the pretraining and posttraining recording sessions.
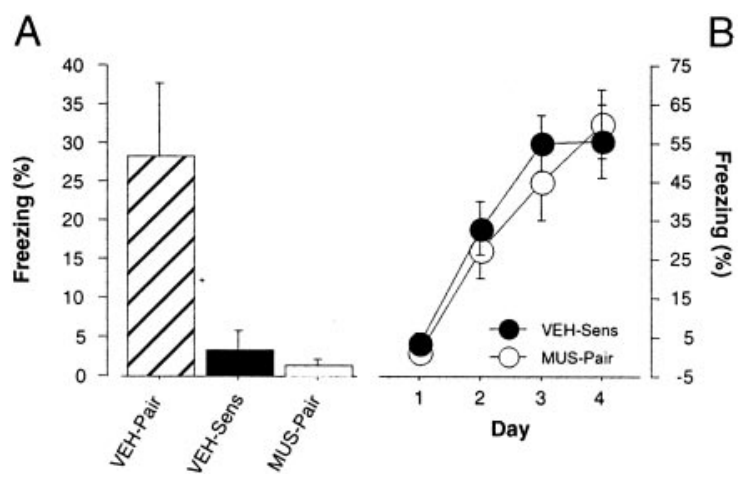

Figure 4. Reacquisition of conditional freezing after training under muscimol inactivation of the BLA. $A$, Mean $( \pm$ SEM) percentage of freezing to the white-noise CS during the posttraining test in VEH-Pair (hatched bars), VEH-Sens (filled bars), and MUS-Pair (open bars). B, Mean $( \pm$ SEM) percentage of freezing to the white-noise CS during $4 \mathrm{~d}$ of reacquisition training (one CS-US trial per day) in VEH-Sens $(-)$ and MUS-Pair (O). Note that the data plotted for Day 1 are the same as those shown in $A$. In this experiment, freezing behavior was measured during a $60 \mathrm{sec}$ white-noise CS on each of the reacquisition days. This allowed us to quantify CS-elicited freezing during the daily CS-US trial.

different rates after training under the conditions described above. Additional rats (24) were assigned to three groups $(n=8$ per group): VEH-Pair, MUS-Pair, and VEH-Sens. The groups were treated identically to those described above, except that recording electrodes were not implanted in MGN. Moreover, subjects received reacquisition training consisting of a single white-noise (60 sec, $85 \mathrm{~dB})$-footshock $(0.5 \mathrm{sec}, 1.0 \mathrm{~mA})$ trial each day for $4 \mathrm{~d}$ after the initial posttraining extinction test (two $40 \mathrm{~min}$ context extinction sessions were interposed between each tone reacquisition session to limit freezing to the contextual cues of the chamber).

As shown in Figure $4 A$, we replicated the deficit in the acquisition of conditional freezing in rats trained after infusion of muscimol into the BLA $\left[F_{(2,21)}=6.7, p<0.01\right]$. More importantly, we found that there was no difference in the rates of reacquisition of auditory fear conditioning in MUS-Pair and $\mathrm{VEH}-$ Sens rats during the second phase of reacquisition training (Fig. $4 B)\left[F_{(3,42)}<1\right]$. Thus, training under muscimol inactivation of the BLA did not yield savings of the auditory fear memory. This suggests that the small elevation in CS-elicited unit activity in MGN after auditory fear conditioning is not sufficient to support conditional fear responses, at least in the case of freezing behavior. 


\section{DISCUSSION}

The present results provide important new insight into the role of the BLA in the development of associative neuronal plasticity in the MGN during auditory fear conditioning. Reversible inactivation of the BLA with muscimol during fear conditioning produced a marked impairment in the development of both conditioning-related increases in MGN spike firing and conditional freezing behavior. Moreover, rats trained under BLA inactivation did not exhibit savings of fear memory during reacquisition training. Together with the results of Poremba and Gabriel (2001), these data indicate that bidirectional interactions between the amygdala and thalamus are essential for the development of associative neuronal plasticity in these structures during aversive learning.

What are the implications of these data for current theories of amygdaloid function in fear conditioning? Clearly, these results are consistent with the proposed role for the BLA in encoding CS-US associations during fear conditioning (Davis, 1994; Fanselow and LeDoux, 1999; Maren, 1999a). By this view, CS-US association in the BLA results in increases in CS-elicited neuronal firing in BLA neurons during fear conditioning. Associative neuronal plasticity in the BLA may then be passively mirrored in the MGN or may trigger events in the MGN that foster the development of neuronal plasticity in the thalamus. However, these data do not support the view that the MGN is the necessary and sufficient locus for CS-US association during fear conditioning. By this view, the amygdala serves to generate conditional responses and modulate cortical storage, processes that are held to depend on the development of associative neuronal plasticity in the MGN (Weinberger, 1998). In fact, neuronal plasticity in the MGN appears to depend on the amygdala.

Although we propose a parsimonious view that associative coding in the BLA is necessary for conditioning-related spike firing in the MGN, it remains possible that BLA is engaged in a nonassociative process that triggers plasticity in MGN. Activation of BLA neurons by footshock USs, for example, may initiate the encoding of CS-US associations in the MGN (Shors and Mathew, 1998; Poremba and Gabriel, 2001). The fact that shock stress facilitates Pavlovian conditioning via an amygdaladependent mechanism is consistent with this possibility (Shors and Mathew, 1998). By this view, then, the amygdala is not itself a locus for the encoding or storage of fear memories but is essential for initiating these processes elsewhere in the brain (McGaugh, 2000). Further studies are required to differentiate these possibilities. In either case, the BLA must gain access to the MGN; this may involve a disynaptic pathway through the auditory cortex (the BLA does not project directly to the MGN).

Recently, it has been reported that the BLA is not required for the development of short-latency (within $50 \mathrm{msec}$ of CS onset) neuronal plasticity in the auditory cortex (Armony et al., 1998). Indeed, conditioning-related plasticity in the BLA and auditory cortex appears to be independent. For example, amygdaloid plasticity precedes that in the cortex during training (Quirk et al., 1997) and appears to develop in thalamo-amygdala projections (Quirk et al., 1995, 1997; Maren, 2000). Thus, these data suggest that parallel memory traces develop in the BLA and auditory cortex during auditory fear conditioning. The present study, however, raises questions concerning the behavioral relevance of fear conditioning-induced neuronal plasticity in the auditory cortex. If neuronal plasticity develops in the auditory cortex of rats condi- tioned under BLA inactivation (as in the present study), then it is apparently not sufficient to generate conditional freezing or behavioral savings (both of which were absent in MUS-Pair rats). This suggests that the ability of CSs to elicit conditional freezing depends on neuronal plasticity in the BLA, not the auditory cortex. It may be the case that the cortical plasticity supports other "cognitive" memories for fear conditioning that direct fear responses, such as avoidance, in animals with amygdala lesions (Vazdarjanova and McGaugh, 1998). Indeed, it has been reported that humans with amygdala damage report normal explicit memories for auditory fear conditioning, despite robust deficits in autonomic conditional responses (Bechara et al., 1995).

In conclusion, the present results reveal that BLA neurons are essential for the development of neuronal plasticity in the MGN during auditory fear conditioning in rats. These results complement and extend those recently obtained by Poremba and Gabriel (2001) in an instrumental avoidance conditioning task in rabbits, which also requires circuitry in the MGN and BLA (Poremba and Gabriel, 1997a,b). Because both instrumental avoidance and auditory fear conditioning require the acquisition of Pavlovian CS-US associations, our results converge on the common conclusion that the BLA is importantly involved in encoding CS-US associations during aversive conditioning. Further studies are required to understand the routes and mechanisms by which the BLA influences the induction and expression of neuronal plasticity in other brain areas.

\section{REFERENCES}

Armony JL, Quirk GJ, LeDoux JE (1998) Differential effects of amygdala lesions on early and late plastic components of auditory cortex spike trains during fear conditioning. J Neurosci 18:2592-2601.

Bechara A, Tranel D, Damasio H, Adolphs R, Rockland C, Damasio AR (1995) Double dissociation of conditioning and declarative knowledge relative to the amygdala and hippocampus in humans. Science 269:1115-1118

Bordi F, LeDoux JE (1994) Response properties of single units in areas of rat auditory thalamus that project to the amygdala. I. Acoustic discharge patterns and frequency receptive fields. Exp Brain Res 98:261-274.

Cahill L, Weinberger NM, Roozendaal B, McGaugh JL (1999) Is the amygdala a locus of "conditioned fear"? Some questions and caveats. Neuron 23:227-228.

Davis M (1994) The role of the amygdala in emotional learning. Int Rev Neurobiol 36:225-266.

Edeline JM, Weinberger NM (1992) Associative retuning in the thalamic source of input to the amygdala and auditory cortex: receptive field plasticity in the medial division of the medial geniculate body. Behav Neurosci 106:81-105.

Fanselow MS, LeDoux JE (1999) Why we think plasticity underlying Pavlovian fear conditioning occurs in the basolateral amygdala. Neuron 23:229-232.

Gabriel M, Saltwick SE, Miller JD (1975) Conditioning and reversal of short-latency multiple-unit responses in the rabbit medial geniculate nucleus. Science 189:1108-1109.

Gerren RA, Weinberger NM (1983) Long-term potentiation in the magnocellular medial geniculate nucleus of the anesthetized cat. Brain Res 265:138-142.

LeDoux JE (2000) Emotion circuits in the brain. Annu Rev Neurosci 23:155-184.

LeDoux JE, Farb C, Ruggiero DA (1990) Topographic organization of neurons in the acoustic thalamus that project to the amygdala. J Neurosci 10:1043-1054.

Maren S (1999a) Long-term potentiation in the amygdala: a mechanism for emotional learning and memory. Trends Neurosci 22:561-567.

Maren S (1999b) Neurotoxic basolateral amygdala lesions impair learning and memory but not the performance of conditional fear in rats. J Neurosci 19:8696-8703.

Maren S (2000) Auditory fear conditioning increases CS-elicited spike firing in lateral amygdala neurons even after extensive overtraining. Eur J Neurosci 12:4047-4054.

McEchron MD, Green EJ, Winters RW, Nolen TG, Schneiderman N, McCabe PM (1996) Changes of synaptic efficacy in the medial genic- 
ulate nucleus as a result of auditory classical conditioning. J Neurosci 16:1273-1283.

McGaugh JL (2000) Memory: a century of consolidation. Science 287:248-251.

Poremba A, Gabriel M (1997a) Amygdalar lesions block discriminative avoidance learning and cingulothalamic training-induced neuronal plasticity in rabbits. J Neurosci 17:5237-5244.

Poremba A, Gabriel M (1997b) Medial geniculate lesions block amygdalar and cingulothalamic learning-related neuronal activity. J Neurosci 17:8645-8655.

Poremba A, Gabriel M (2001) Amygdalar efferents initiate auditory thalamic discriminative training-induced neuronal activity. J Neurosci $21: 260-268$.

Quirk GJ, Repa C, LeDoux JE (1995) Fear conditioning enhances shortlatency auditory responses of lateral amygdala neurons: parallel recordings in the freely behaving rat. Neuron 15:1029-1039.

Quirk GJ, Armony JL, LeDoux JE (1997) Fear conditioning enhances different temporal components of tone-evoked spike trains in auditory cortex and lateral amygdala. Neuron 19:613-624.

Shors TJ, Mathew PR (1998) NMDA receptor antagonism in the basolateral but not central nucleus of the amygdala prevents the induction of facilitated learning in response to stress. Learn Mem 5:220-230.

Supple Jr W, Kapp BS (1989) Response characteristics of neurons in themedial component of the medial geniculate nucleus during Pavlovian differential fear conditioning in rabbits. Behav Neurosci 103:1276-1286.

Swanson LW (1998) Brain maps: structure of the rat brain, Ed 2. Amsterdam: Elsevier.

Vazdarjanova A, McGaugh JL (1998) Basolateral amygdala is not critical for cognitive memory of contextual fear conditioning. Proc Natl Acad Sci USA 95:15003-15007.

Weinberger NM (1998) Physiological memory in primary auditory cortex: characteristics and mechanisms. Neurobiol Learn Mem 70: 226-251. 